\title{
The Assessment of 5 Polymorphisms in the USP9Y Gene in Infertile Patients with Non-obstructive Azoospermia
}

\author{
Naser Kalhor (iD) ${ }^{1}$, Hoda Fazaeli ${ }^{1}$, Azar Sheikholeslami ${ }^{1}$ and Faezeh Davoodi Asl (iD ${ }^{1,{ }^{*}}$ \\ ${ }^{1}$ Department of Mesenchymal Stem Cells, Academic Centre for Education, Culture and Research, Qom Branch, Qom, Iran \\ "Corresponding author: Department of Mesenchymal Stem Cells, Academic Centre for Education, Culture and Research, Qom Branch, Qom, Iran. Email: \\ faeze.davoodi64@yahoo.com
}

Received 2020 September 12; Revised 2020 December 24; Accepted 2021 January 03.

\begin{abstract}
Background: Male factor infertility that is the cause of about half of the infertility cases, may occur due to azoospermia. Because spermatogenesis defects may lead to non-obstructive azoospermia (NOA), investigating the factors involved in spermatogenesis, including hormones and genes, is one of the most important aspects in understanding the mechanism of NOA in men.

Objectives: Male infertility is a complex disorder that affects a large proportion of men, yet many of its causes are unknown. Clarifying its genetic basis may help to identify the causes of infertility and provide effective treatment for patients.

Methods: In this case-control study, single nucleotide polymorphisms (SNPs) located in the USP9Y gene were investigated. Accordingly, 100 healthy men and 100 NOA patients were regarded as control and case groups, respectively. Testis tissue samples were taken during the testicular sperm extraction (TESE) procedure, DNA extraction was done, and ARMS PCR was designed and performed. The rs3212292, rs2032597, rs2032604, rs2032598, and rs717268 polymorphisms in the USP9Y gene were analyzed by Tetra-ARMS PCR. Furthermore, the serum levels of sex hormones were assessed by the ELISA technique. Finally, the obtained data were analyzed by SPSS software.

Results: According to the obtained results, there was no significant difference in case of genotype frequency of investigated SNPs between the case and control groups $(\mathrm{P} \geq 0.05)$. The mean FSH and LH levels in the control group were relatively lower than the case group $(\mathrm{P} \leq 0.05)$, whereas no significant difference in the mean testosterone level was observed between the two groups $(\mathrm{P} \geq$ 0.05).

Conclusions: Polymorphisms of the assessed SNPs showed no effect on the frequency of azoospermia; Thus, polymorphisms did not increase the risk of NOA and also did not have a protective effect against the disease. Also, the results showed that the serum levels of FSH and LH increased in NOA patients.
\end{abstract}

Keywords: Male Infertility, Non-obstructive Azoospermia, Single Nucleotide Polymorphisms, USP9Y, ARMS PCR

\section{Background}

Azoospermia is one of the most serious reproductive disorders that occurs in $1 \%$ of the male population. Various factors are involved in azoospermia; however, its precise etiology is almost unknown (1). Most of these factors are complex gene-environment interactions (2). This reproductive-related disorder is classified as obstructive azoospermia (OA), induced by ejaculatory pathway obstruction, and non-obstructive azoospermia (NOA) caused by a failure in spermatogenesis (3). NOA is a disorder of genetic origin that is represented as a failure of spermatogenesis within the testis, including various reasons, such as chromosomal abnormalities and Y chromosome microdeletions (4). This phenotype has different causes (affecting various functions, such as differentiation of gonads, the activity of the hypothalamic-pituitary axis, and spermatogenesis), making it a complex and heterogeneous disorder (5). Essential genes involved in spermatogenesis are placed on the proximal area of the Y chromosome (Yp11). Azoospermia factor (AZF) is located in this region, which includes sub-regions of AZFa, AZFb, and AZFC (6). Ubiquitin-specific peptidase 9, Y-link (USP9Y), is a functional gene on the AZFa region with a DNA of $170 \mathrm{~kb}$ and at least 46 exons. USP9Y is responsible for encoding a protein (7) that acts as ubiquitin C-terminal hydrolase, which its elimination is related to azoospermia or violent oligospermia (8). USP9Y expression is limited to spermatids, and point mutations in this gene can result in various clinical phenotypes of male infertility, including spermatid maturation arrest, oligospermia, or asthenozoospermia, linked to decreased sperm cell mobility and concentration (9). Also, complete loss of the gene is associated with 
NOA $(10,11)$. In many complex diseases, genetic susceptibilities have been identified through genome-wide association studies (GWAS) using single-nucleotide polymorphism (SNP) arrays $(12,13)$. There is not enough information about the relationship between the USP9Y gene polymorphisms and azoospermia; thus, this study aimed at detecting the relationship between 5 polymorphisms of USP9Y and azoospermia susceptibility in the target population.

\section{Objectives}

Because different genes are responsible for the process of spermatogenesis and the formation of azoospermia, it is important to evaluate the genetic basis of infertility. In this study, we focused on the probable communication between the polymorphisms in the USP9Y gene and NOA.

\section{Methods}

This case-control study was approved by the Academic Center for Education, Culture, and Research (ACECR), Qom province, and the Biomedical Research Ethics Committee (IR.ACECR.JDM.REC.1397.015).

Regarding the azoospermia frequency in the population (1\%), and the earlier findings, the minimum sample size was calculated based on the following formula (14):

$$
\frac{\left(Z_{1-\frac{a}{2}}\right)^{2} p(1-p)}{d^{2}}
$$

Accordingly, 200 men attending the infertility treatment center from August 2016 to August 2017 were divided into the case (NOA) and control groups, and written informed consent was obtained before enrolling in the study. The inclusion criteria were as follows: Participants aged 25 - 35 years old and being physically and anatomically healthy with normal karyotype (Table 1 ). Healthy people for the control group had at least one healthy child with no more than two years passing since the birth of the last child. Semen from both cases and controls were analyzed according to WHO protocol, and urological examinations were performed for anatomical integrity of the genital system. Also, the NOA of the cases was approved by an urologist. Patients with anatomic disorders of genitalia, testis neoplasms, chromosomal numerical and structural abnormalities, or Y chromosome microdeletions were excluded.

In order to confirm NOA, testicular biopsies of patients who had no sperm according to their semen analysis were assessed by a pathologist. Furthermore, serum levels of $\mathrm{LH}, \mathrm{FSH}$, and testosterone were measured using an ELISA kit in the case and control groups.

\begin{tabular}{lc}
\hline Table 1. Inclusion Criteria for Non-obstructive Azoospermic Men & \\
\hline Karyotype & $\mathbf{4 6 , \mathbf { X Y }}$ \\
\hline Microdeletion chromosome Y & NO \\
Spermogram with centrifuge & No sperm \\
TESE & No sperm \\
\hline Age (y) & $25-35$ \\
\hline Varicocele & No \\
\hline Gonadotropins (chemotherapy/radiation) & No \\
\hline
\end{tabular}

Genomic DNA was extracted from blood samples using GeneAll $®$ ExgeneTM kit. We used the tetra-primer amplification refractory mutation system- polymerase chain (PCR) method, in which two pairs of primers are used to analyze USP9Y rs2032597 (A>C), rs2032604 (T>G), rs2032598 (T>C), rs717268 (G>A), and rs3212292 ( $>$ >A) genotypes. Primers were designed by Primer1 online software (http://primer1.soton.ac.uk/primer1 .html). PCR primers are given in Table 2 .

Statistical analysis for genotype and allele frequencies, azoospermia association, and hormonal evaluation were performed by the $\chi^{2}$ and paired $t$-test using SPSS software. A P-value of less than 0.05 was considered to be statistically significant.

\section{Results}

The mean age of the control and case subjects was 31.12 \pm 1.38 and $31 \pm 1.688$ years, respectively. Table 3 presents the frequencies of genotypes and alleles in 5 SNPs. The $\chi^{2}$ test showed that there was no significant difference between the genotypic and allelic frequencies between the case and control groups, and these genotypes did not increase the chance of disease or showed no protective effect against NOA. The electrophoresis results of 5 SNPs are shown in Figure 1.

The mean FSH level in the control and case group was $7.43 \pm 1.947$ and $16.88 \pm 2.188 \mathrm{mIU} / \mathrm{mL}$, respectively. The Mann-Whitney U test showed that the observed mean difference was significant $(\mathrm{P}<0.001)$, and the FSH hormone increased in cases. The mean LH level was $5.7 \pm 1.828$ and $12.18 \pm 1.471 \mathrm{mIU} / \mathrm{ml}$ in the control and case groups, respectively, and the observed difference between the two groups was significant $(\mathrm{P}=0.002)$. The mean testosterone level in the control and case group was $4.6 \pm 1.262$ and $4.83 \pm$ $1.292 \mathrm{ng} / \mathrm{dL}$, respectively. The independent $t$-test indicated that the difference in mean testosterone level observed between the two groups was not significant $(\mathrm{P}=0.849)$ (Table 4). 


\begin{tabular}{|c|c|c|}
\hline Rs & Nucleotide Sequence & PCR Product (bp) \\
\hline rs3212292 & & $\mathrm{FO}-\mathrm{RO}=463 ; \mathrm{FO}-\mathrm{RT}=296 ; \mathrm{RO}-\mathrm{FA}=221$ \\
\hline FA (A allele) & 339 GCTTGGGATTTTTCTCCTGGACATCA 364 & \\
\hline RT (T allele) & 391 ACCTTAAAGCAATCAAAAAGATGATGAA 364 & \\
\hline $\mathrm{FO}\left(5^{\prime}-3^{\prime}\right)$ & 96 TGCCAGGTATTAAATGACAGTTCTAAAGCA 125 & \\
\hline $\mathrm{RO}\left(5^{\prime}-3^{\prime}\right)$ & 558 TGATTGGAAGAAACAACAATGACCAATT 531 & \\
\hline rs2032597 & & $\mathrm{FO}-\mathrm{RO}=441 ; \mathrm{FO}-\mathrm{RC}=289 ; \mathrm{RO}-\mathrm{FA}=208$ \\
\hline FA (A allele) & 496 TTTGTTAAATAATTTCATGTTTGTCCA 522 & \\
\hline RC (C allele) & 550 ACATAACTTAATAAGAGCTGCAATTACTG 522 & \\
\hline Forward outer primer ( $\left.5^{\prime}-3^{\prime}\right)$ & 262 TAAATTTTCCATTTCTAGTATGCTTCAC 289 & \\
\hline Reverse outer primer $\left(5^{\prime}-3^{\prime}\right)$ & 702 AAAAGAACTAAACTTGCCAATTACTTTC 675 & \\
\hline rs2032598 & & $\mathrm{FO}-\mathrm{RO}=318 ; \mathrm{FO}-\mathrm{RT}=174 ; \mathrm{RO}-\mathrm{FC}=197$ \\
\hline FC (C allele) & 331 ATAATGGCCAGCAATTTAGTATTGACC 357 & \\
\hline RT (T allele) & 382 AACAGCACATGCATTAGTAAAAGGCA 357 & \\
\hline Forward outer primer $\left(5^{\prime}-3^{\prime}\right)$ & 209 AAAGAAAAGGCTCTTACATTACAGGACC 236 & \\
\hline Reverse outer primer (5' - $\left.3^{\prime}\right)$ & 526 GAGCAAGATTCCATCTAAAAACAAAACA 499 & \\
\hline rs2032604 & & $\mathrm{FO}-\mathrm{RO}=505 ; \mathrm{FO}-\mathrm{RG}=\mathbf{2 6 5} ; \mathrm{RO}-\mathrm{FT}=296$ \\
\hline FT (T allele) & 493 TATCCCCCAAACCCATTTTGATGCCTT 519 & \\
\hline RG (G allele) & 547 GAAAATAATAATTGAAGACCTTTTAAATC 519 & \\
\hline Forward outer primer ( $\left.5^{\prime}-3^{\prime}\right)$ & 252 AAGATGTTAAAGAGGCCAGCTTTGTG 277 & \\
\hline Reverse outer primer ( $\left.5^{\prime}-3^{\prime}\right)$ & 756 GGTTACAAAGAACATATCACAATGGCAA 729 & \\
\hline rs717268 & & $\mathrm{FO}-\mathrm{RO}=373 ; \mathrm{FO}-\mathrm{RA}=204 ; \mathrm{RO}-\mathrm{FG}=226$ \\
\hline FG (G allele) & 234 ATAGGATGTATTATTTTCTATGATTCTGAG 263 & \\
\hline RA (A allele) & 289 TGTTCCTTTACATATGAGATGTATGTT 263 & \\
\hline Forward outer primer $\left(5^{\prime}-3^{\prime}\right)$ & 86 AACTTCTGAGTGAATTTCTTAGTTGTAG 113 & \\
\hline Reverse outer primer ( $\left.5^{\prime}-3^{\prime}\right)$ & 458 CATAGTACTCAGATGCTGATTATACAAA 431 & \\
\hline
\end{tabular}

\section{Discussion}

Infertility is a multifactorial issue in which various factors, such as the environment and genetics, can be influential. Studies have shown that $50 \%$ of infertilities are related to men. The most important factors in male infertility are genetic factors that affect important physiological processes, such as hormonal homeostasis, spermatogenesis, and sperm quality (15). About 200 genes have been found so far that can regulate spermatogenesis, 30 of which are on the Y chromosome. Two major genetic factors in men's infertilities are $\mathrm{Y}$ chromosome microdeletions and chromosomal abnormalities $(16,17)$. Around $25 \%$ of NOA cases can be explained by single genetic abnormalities (including chromosome aberrations and point mutations), with the remaining patients considered idiopathic. It has been indicated that idiopathic NOA has a multifactorial etiology, in which both environmental and genetic factors may con- tribute to the development of the disease. In these cases, wide variations in the human genome, mainly single nucleotide polymorphisms (SNPs) and copy number variants (CNVs), are likely to confer genetic predisposition, complicating the elucidation of the underlying pathological mechanisms $(18,19)$. According to the influential advances in infertility treatment using assisted reproductive techniques (ART), which increase the likelihood of transferring genetic abnormalities to the next generations, identification of genetic abnormalities sounds valuable (20). In fact, the frequency of male infertility varies among different geographical regions and ethnic groups. It was found that among azoospermia cases, $83.7 \%$ were due to NOA(21). Also, Fogle et al. showed that 93\% of azoospermia cases were recorded as NOA (22). However, the most geographical and ethnic reports in male infertility are on Y chromosome microdeletions.

Malekasgar and Masoudi showed that 51.6\% of 


\begin{tabular}{|c|c|c|c|c|}
\hline rs/Genotype \& Allele & Non-obstructive Azoospermia, No. (\%) & Control, No. (\%) & P-Value & OR $(95 \% \mathrm{CI})$ \\
\hline $\operatorname{rs3212292}(T>A)$ & & & 0.346 & $1.568(0.612-4.019)$ \\
\hline A & $12(12)$ & $8(8)$ & & \\
\hline $\mathrm{T}$ & $88(88)$ & $92(92)$ & & \\
\hline $\operatorname{rs2032597}(A>C)$ & & & 0.744 & $0.899(0.474-1.705)$ \\
\hline A & $76(76)$ & $74(74)$ & & \\
\hline $\mathrm{C}$ & $24(24)$ & $26(26)$ & & \\
\hline $\operatorname{rs2032598}(T>C)$ & & & 0.313 & $1.405(0.724-2.728)$ \\
\hline $\mathrm{C}$ & $26(26)$ & $20(20)$ & & \\
\hline $\mathrm{T}$ & $74(74)$ & $80(80)$ & & \\
\hline $\operatorname{rs2032604}(T>G)$ & & & 0.192 & $1.784(0.742-4.292)$ \\
\hline G & $15(15)$ & $9(9)$ & & \\
\hline $\mathrm{T}$ & $85(85)$ & $91(91)$ & & \\
\hline $\operatorname{rs717268}(G>A)$ & & & 0.39 & $1.652(0.521-5.236)$ \\
\hline A & $8(8)$ & $5(5)$ & & \\
\hline G & $92(92)$ & $95(95)$ & & \\
\hline
\end{tabular}

\section{A}
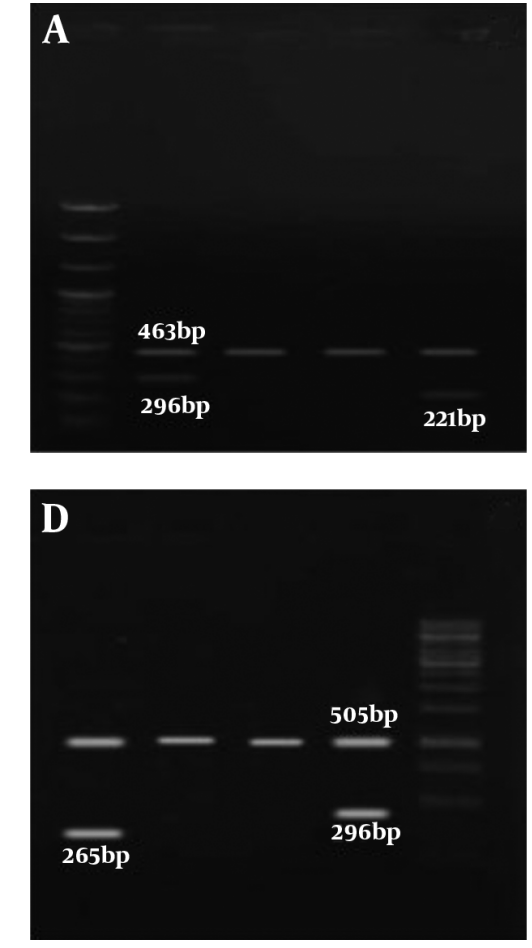
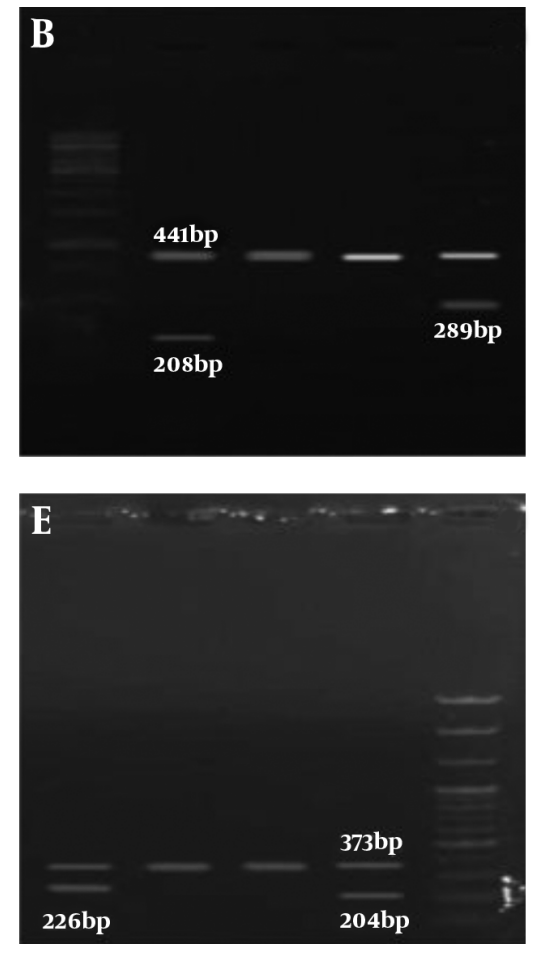

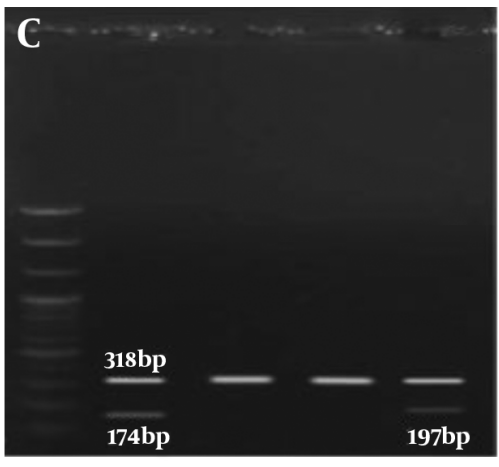

F

1. rs3212292 (A allele 221 bp. T allele 296 bp) 2. rs2032597 (A allele 208 bp. C allele 289 bp) 3. rs2032598 (C allele 197 bp. T allele 174 bp) 4. rs2032604 (T allele 265 bp. G allele 296 bp) 5. rs717268 ( $G$ allele 226 bp. A allele 204 bp) Ladder 100 


\begin{tabular}{lccc}
\hline Table 4. Comparison of Hormonal Levels in the Control and Non-obstructive Azoospermic Men & & \\
\hline Hormone & Control & Non-obstructive Azoospermia & P-Value \\
\hline FSH & $7.43 \pm 1.95$ & $16.88 \pm 2.19$ & $<0.0001$ \\
LH & $5.7 \pm 1.82$ & $12.18 \pm 1.47$ \\
Testosterone & $4.6 \pm 1.26$ & $4.83 \pm 1.29$ \\
\hline
\end{tabular}

azoospermic infertile men and $52.6 \%$ of severe oligozoospermic patients from north Iran carry Y chromosome microdeletions $(23,24)$. In the Iranian population, the $Y$ microdeletion frequency, according to the databases from 2003 - 2012, has shown variable ranges from 5 - 52\%, which is within the worldwide range (15). This wide range of variability may be the consequence of different factors, such as the sample size, type of patient selection, and the variances among the populations (25). Also, Omrani et al. reported $24 \%$ microdeletions in infertile men from West Azarbayjan (26). It should be noticed that our study was performed on 200 participants referring to the infertility treatment center of ACECR in Qom province. One of the genes involved in the spermatogenesis process is the USP9Y gene, which was selected to examine in this study. Deletions affecting USP9Y were initially highly associated with mild to severe azoospermia or oligozoospermia (27). It was later documented that partial deletion of USP9Y in men is correlated with the milder infertility phenotype, indicating a minor effect for USP9Y in spermatogenesis (28). A study by Sun et al. on 576 infertile men and 96 healthy men showed that single-gene deletion associated with spermatogenic insufficiency occurred in USP9Y (29). Thus, USP9Y is not required for normal spermatogenesis, but its effects on fertility may be effective in combination with other genes in the AZFa region (30). In 2000, Foresta et al. analyzed 173 infertile men focusing on the AZFa region and found deletions in 9 patients, of whom one case showed deletions in the USP9Y gene (31). There is contradictory evidence about the impact of this gene on infertility and spermatogenesis, and the association between polymorphisms in the USP9Y gene and azoospermia has also not been investigated. We tested 5 polymorphisms of the USP9Y gene in this study. Some SNPs of this gene have been evaluated and shown to be associated with some other diseases. In a study, Wang and Park showed that the risk of schizophrenia, autism, and prostate cancer could be increased by rs2032598 and rs2032604 SNPs; however, no population-based study on azoospermia has yet been performed $(32,33)$.

It is of particular interest that the ubiquitin protein encoded by the USP9Y gene plays an important role in infertility and can affect the polymorphism of the USP9Y gene in male infertilities $(34,35)$. Studies have shown that the levels of sex hormones, such as LH, FSH, and testosterone were significantly higher in infertile men in comparison with healthy men. These hormonal changes were much more seen in azoospermic men than in other infertile subgroups $(36,37)$. These results suggest that the FSH, LH, prolactin, and testosterone levels cannot predict microdeletions in AZF in infertile men. More studies with a larger sample size should be considered to assess these microdeletions (38). Zhao et al. stated that sperm density, total sperm count, number of motile sperm, and percentage of motile sperm were positively associated with the testicular volume (39). However, our results in this study showed a decrease in FSH and LH hormones in the case group compared with the control group. Also, testosterone hormone levels in the case and control groups did not show a significant difference.

\subsection{Conclusion}

In general, our results showed that in the studied population, the frequency of genotypes and alleles did not play a role in developing NOA. The results of this study showed that one of the important reasons for NOA can be hormonal disorders so that in NOA, the levels of FSH and LH hormones are increased, which indicates that in people with sperm disorders, hormone levels should be checked at first, and then other tests and more detailed investigations should be regarded.

\section{Acknowledgments}

The authors thank the Academic Center for Education, Culture, and Research (ACECR) for funding this Research.

\section{Footnotes}

Authors' Contribution: Study concept and design: N.K., and F.D.; Analysis and interpretation of data: N.K. and F.D.; Drafting of the manuscript: H.F., and A.Sh.; Critical revision of the manuscript: F.D., A.Sh.; Statistical analysis: N.K.

Conflict of Interests: The authors declared no conflict of interest

Ethical Approval: IR.ACECR.JDM.REC.1397.6

Funding/Support: The authors appreciate funding support from the Academic Centre for Education, Culture and Research, Qom Branch, Qom, Iran. 


\section{References}

1. Agarwal A, Mulgund A, Hamada A, Chyatte MR. A unique view on male infertility around the globe. Reprod Biol Endocrinol. 2015;13:37. doi: 10.1186/s12958-015-0032-1. [PubMed: 25928197]. [PubMed Central: PMC4424520].

2. Cocuzza M, Alvarenga C, Pagani R. The epidemiology and etiology of azoospermia. Clinics (Sao Paulo). 2013;68(S1):15-26. doi: 10.6061/clinics/2013(sup01)03. [PubMed: 23503951]. [PubMed Central: PMC3583160].

3. Esteves SC. Clinical management of infertile men with nonobstructive azoospermia. Asian J Androl. 2015;17(3):459. doi: 10.4103/1008-682X.148719. [PubMed Central: PMC4430952].

4. Nagvenkar P, Desai K, Hinduja I, Zaveri K. Chromosomal studies in infertile men with oligozoospermia \& non-obstructive azoospermia. Indian J Med Res. 2005;122(1):34-42. [PubMed: 16106088].

5. Cervan-Martin M, Castilla JA, Palomino-Morales RJ, Carmona FD. Genetic landscape of non-obstructive azoospermia and new perspectives for the clinic. J Clin Med. 2020;9(2). doi: 10.3390/jcm9020300. [PubMed: 31973052]. [PubMed Central: PMC7074441].

6. Kent-First M, Muallem A, Shultz J, Pryor J, Roberts K, Nolten W, et al. Defining regions of the Y-chromosome responsible for male infertility and identification of a fourth AZF region (AZFd) by Ychromosome microdeletion detection. Mol Reprod Dev. 1999;53(1):2741. doi: 10.1002/(SICI)1098-2795(199905)53:1<27::AID-MRD4>3.0.CO;2W. [PubMed: 10230814].

7. Metwally BAI. Y chromosome microdeletions in husbands of women with recurrent unexplanied first trimester abortions [master's thesis]. Cairo, Egypt: Ain Shams University; 2010.

8. Lee JY, Dada R, Sabanegh E, Carpi A, Agarwal A. Role of genetics in azoospermia. Urology. 2011;77(3):598-601. doi: 10.1016/j.urology.2010.10.001. [PubMed: 21195467].

9. Nuti F, Krausz C. Gene polymorphisms/mutations relevant to abnormal spermatogenesis. Reprod Biomed Online. 2008;16(4):504-13. doi: 10.1016/s1472-6483(10)60457-9. [PubMed: 18413059].

10. Tyler-Smith C, Krausz C. The will-o'-the-wisp of genetics-hunting for the azoospermia factor gene. $N$ Engl J Med. 2009;360(9):925-7. doi: 10.1056/NEJMe0900301. [PubMed: 19246366]. [PubMed Central: PMC2700876].

11. Vogt PH. Azoospermia factor (AZF) in Yq11: Towards a molecular understanding of its function for human male fertility and spermatogenesis. Reprod Biomed Online. 2005;10(1):81-93. doi: 10.1016/s14726483(10)60807-3. [PubMed:15705299].

12. Aston KI. Genetic susceptibility to male infertility: News from genome-wide association studies. Andrology. 2014;2(3):315-21. doi: 10.1111/j.2047-2927.2014.00188.x. [PubMed: 24574159].

13. Kassam S, Meyer P, Corfield A, Mikuz G, Sergi C. Single nucleotide polymorphisms (SNPs): History, biotechnological outlook and practical applications. Current Pharmacogenomics. 2005;3(3):237-45. doi: 10.2174/1570160054864021.

14. Charan J, Biswas T. How to calculate sample size for different study designs in medical research? Indian J Psychol Med. 2013;35(2):121-6. doi: 10.4103/0253-7176.116232. [PubMed: 24049221]. [PubMed Central: PMC3775042].

15. Moghbelinejad S, Mozdarani H, Ghoraeian P, Asadi R. Basic and clinical genetic studies on male infertility in Iran during 2000-2016: A review. Int J Reprod Biomed. 2018;16(3):131-48. [PubMed: 29766145]. [PubMed Central: PMC5944436].

16. Ferlin A, Raicu F, Gatta V, Zuccarello D, Palka G, Foresta C. Male infertility: Role of genetic background. Reprod Biomed Online. 2007;14(6):73445. doi: 10.1016/s1472-6483(10)60677-3. [PubMed: 17579990].

17. Van Assche E, Bonduelle M, Tournaye H, Joris H, Verheyen G, Devroey P, et al. Cytogenetics of infertile men. Hum Reprod. 1996;11 Suppl 4:1-26. doi:10.1093/humrep/11.suppl_4.1. [PubMed: 9147109].

18. Krausz C, Riera-Escamilla A. Monogenic forms of male infertility. Exp Suppl. 2019;111:341-66. doi: 10.1007/978-3-030-25905-1_16. [PubMed: 31588539].
19. Tournaye H, Krausz C, Oates RD. Novel concepts in the aetiology of male reproductive impairment. Lancet Diabetes Endocrinol. 2017;5(7):544-53. doi: 10.1016/S2213-8587(16)30040-7. [PubMed: 27395771].

20. Ahmadi Rastegar D, Sharifi Tabar M, Alikhani M, Parsamatin P, Sahraneshin Samani F, Sabbaghian M, et al. Isoform-level gene expression profiles of human $Y$ chromosome azoospermia factor genes and their X chromosome paralogs in the testicular tissue of nonobstructive azoospermia patients. J Proteome Res. 2015;14(9):3595605. doi: 10.1021/acs.jproteome.5b00520. [PubMed: 26162009].

21. Laan M. Systematic review of the monogenetic causes of male infertility: The first step towards diagnostic gene panels in the andrology clinic. Hum Reprod.2019;34(5):783-5. doi:10.1093/humrep/dez024. [PubMed: 30865269].

22. Rabani SMR, Moosavizadeh A. [A study on causes of azoospermia in urology clinic of Yasuj university of medical sciences]. Armaghane Danesh. 2006;11(3):81-7. Persian.

23. Malekasgar AM, Mombaini H. Screening of ' $\mathrm{Y}$ ' chromosome microdeletions in Iranian infertile males. J Hum Reprod Sci. 2008;1(1):2-9. doi: 10.4103/0974-1208.38973. [PubMed: 19562056]. [PubMed Central: PMC2700682].

24. Masoudi R, Mazaheri-Asadi L, Khorasani S. Partial and complete microdeletions of $Y$ chromosome in infertile males from south of Iran. Mol Biol Res Commun. 2016;5(4):247-55. [PubMed: 28261628]. [PubMed Central: PMC5326488].

25. Fogle RH, Steiner AZ, Marshall FE, Sokol RZ. Etiology of azoospermia in a large nonreferral inner-city population. Fertil Steril. 2006;86(1):1979. doi: 10.1016/j.fertnstert.2005.12.047. [PubMed:16753159].

26. Omrani MD, Samadzadae S, Bagheri M, Attar K. Y chromosome microdeletions in idiopathic infertile men from West Azarbaijan. Urol J. 2006;3(1):38-43. [PubMed: 17590851].

27. Brown GM, Furlong RA, Sargent CA, Erickson RP, Longepied G Mitchell M, et al. Characterisation of the coding sequence and fine mapping of the human DFFRY gene and comparative expression analysis and mapping to the Sxrb interval of the mouse Y chromosome of the Dffry gene. Hum Mol Genet. 1998;7(1):97-107. doi 10.1093/hmg/7.1.97. [PubMed: 9384609].

28. Konishi A, Sakushima K, Isobe S, Sato D. First approval of regenerative medical products under the PMD act in Japan. Cell Stem Cell. 2016;18(4):434-5. doi:10.1016/j.stem.2016.03.011. [PubMed: 27058934]

29. Sun C, Skaletsky H, Birren B, Devon K, Tang Z, Silber S, et al An azoospermic man with a de novo point mutation in the Y-chromosomal gene USP9Y. Nat Genet. 1999;23(4):429-32. doi 10.1038/70539. [PubMed: 10581029].

30. Krausz C, Degl'Innocenti S, Nuti F, Morelli A, Felici F, Sansone M, et al. Natural transmission of USP9Y gene mutations: A new perspective on the role of AZFa genes in male fertility. Hum Mol Genet. 2006;15(18):2673-81. doi: 10.1093/hmg/ddl198. [PubMed:16893908].

31. Foresta C, Ferlin A, Moro E. Deletion and expression analysis of AZFa genes on the human Y chromosome revealed a major role for DBY in male infertility. Hum Mol Genet. 2000;9(8):1161-9. doi 10.1093/hmg/9.8.1161. [PubMed: 10767340].

32. Park WT, Kim JK, Park S, Lee SW, Li X, Kim YB, et al. Metabolic profiling of glucosinolates, anthocyanins, carotenoids, and other secondary metabolites in kohlrabi (Brassica oleracea var. gongylodes). J Agric Food Chem. 2012;60(33):8111-6. doi: 10.1021/jf301667j. [PubMed: 22742768]

33. Wang K, Zhang H, Ma D, Bucan M, Glessner JT, Abrahams BS, et al Common genetic variants on 5 p14.1 associate with autism spectrum disorders. Nature. 2009;459(7246):528-33. doi: 10.1038/nature07999. [PubMed: 19404256]. [PubMed Central: PMC2943511].

34. Luddi A, Margollicci M, Gambera L, Serafini F, Cioni M, De Leo V, et al. Spermatogenesis in a man with complete deletion of USP9Y. $N$ Engl J Med. 2009;360(9):881-5. doi: 10.1056/NEJMoa0806218. [PubMed: 19246359].

35. Shah R, Gupta C. Advances in sperm retrieval techniques in azoospermic men: A systematic review. Arab J Urol. 2018;16(1):125-31. doi: 
10.1016/j.aju.2017.11.010. [PubMed: 29713543]. [PubMed Central: PMC5922005].

36. Gholami D, Jafari-Ghahfarokhi H, Nemati-Dehkordi M, Teimori H Y chromosome microdeletions frequency in idiopathic azoospermia, oligoasthenozoospermia, and oligospermia. Int $J$ Reprod Biomed. 2017;15(11):703-12. [PubMed: 29404532]. [PubMed Central: PMC5780556].

37. Zhang YS, Dai RL, Wang RX, Zhang ZH, Fadlalla E, Liu RZ. Azoospermia factor microdeletions: Occurrence in infertile men with azoospermia and severe oligozoospermia from China. Andrologia. 2014;46(5):535-
40. doi: 10.1111/and.12117. [PubMed: 23701642].

38. Elfateh F, Wang R, Zhang Z, Jiang Y, Chen S, Liu R. Influence of genetic abnormalities on semen quality and male fertility: A fouryear prospective study. Iran J Reprod Med. 2014;12(2):95-102. [PubMed: 24799866]. [PubMed Central: PMC4009560].

39. Zhao LM, Jiang H, Hong K, Huang J, Tang WH, Mao JM, et al. [Successful microdissection testicular sperm extraction in patients with nonmosaic Klinefelter syndrome: Three cases report]. Beijing Da Xue Xиe Bao Yi Xue Ban. 2012;44(4):547-50. Chinese. [PubMed: 22898843]. 